\title{
Cosmic Ray Elemental Spectra and Atmospheric Neutrino Fluxes
}

\author{
Rachel Scrandis, ${ }^{a, *}$ Deven P. Bowman ${ }^{a}$ and Eun-Suk Seo ${ }^{a, b}$ \\ ${ }^{a}$ University of Maryland, Dept. of Physics \\ 4150 Campus Dr, College Park, MD, USA \\ ${ }^{b}$ University of Maryland, Inst. for Phys. Sci. and Tech. \\ 4254 Stadium Dr, College Park, MD, USA \\ E-mail: rscrandi@umd.edu, dbowman3@umd.edu
}

\begin{abstract}
Atmospheric neutrinos are produced when cosmic rays interact with Earth's atmosphere. The relationship between the cosmic ray spectrum and the neutrino spectrum is especially important around the cosmic ray all-particle knee. These energies correspond to the regime in which astrophysical neutrinos begin to dominate the neutrino flux, so accurate modeling of the cosmicray spectrum around the knee can be used to help separate background from signal. Currently, direct measurements of elemental spectra reach their upper energy limit just below the all-particle knee, requiring extrapolation in order to probe the transitional neutrino source energy regime. In this work, the cosmic ray knee is modeled as a transition between acceleration sources, each with a rigidity dependent acceleration limit. Cosmic-ray particles reach the limit at $\mathrm{Z} * \mathrm{E}_{\text {max }}$ where $\mathrm{Z}$ is the particle charge and $\mathrm{E}_{\max }$ is proton's limit. Utilizing the Matrix Cascade Equations code, cosmic-ray elemental spectra were used to calculate resulting atmospheric neutrino fluxes. Various parameterizations to model cosmic rays are explored, the effects of the resulting elemental spectra on the neutrino fluxes are investigated, and the neutrino results are compared to experimental data.
\end{abstract}

$37^{\text {th }}$ International Cosmic Ray Conference (ICRC 2021)

July 12th - 23rd, 2021

Online - Berlin, Germany

\footnotetext{
${ }^{*}$ Presenter
} 


\section{Introduction}

Atmospheric neutrinos are produced when cosmic rays interact with Earth's atmosphere and induce hadronic showers. These neutrinos dominate the measured neutrino spectrum up until $\sim 100$ $\mathrm{TeV}$, above which astrophysical neutrinos begin to separate from the background [1]. It is of interest to characterize the atmospheric neutrino flux, especially at the transition energies from atmospheric to astrophysical, to better analyze potential low energy astrophysical signals. Therefore, in order to gain a better understanding of atmospheric neutrinos, the cosmic rays that produce them are investigated.

Charged cosmic rays are typically protons but also heavier nuclei that are accelerated in astrophysical sources. The all-particle spectrum, spanning many decades of energy, presents a 'knee', or spectral steepening, at around $10^{6} \mathrm{GeV}$. The source of this spectral feature is unknown, although it is widely believed to arise from a change in the underlying cosmic ray accelerators. Furthermore, cosmic rays at knee energies typically produce atmospheric neutrinos at $\sim 100 \mathrm{TeV}$, thus making accurate modeling of this energy range in cosmic rays a key to understanding the atmospheric to astrophysical transition in neutrinos. Such modeling requires information about the underlying spectral composition of the all-particle spectrum, however current direct measurements extend to just below the knee. In this work, we aim to characterize the all-particle knee and neutrino transition by fitting direct and indirect measurement data to various models and calculate expected atmospheric neutrino fluxes using these fits. Our results are then compared to both cosmic ray all-particle data and IceCube's measured atmospheric neutrino spectrum.

\section{Methods}

\subsection{Data Compiled}

In this analysis, cosmic ray elemental spectra measurements from $\sim 10^{9}-10^{14} \mathrm{eV}$ were compiled from direct measurements. These compiled data points are of the 8 most abundant primary particles: proton, $\mathrm{He}, \mathrm{C}, \mathrm{O}, \mathrm{Ne}, \mathrm{Mg}, \mathrm{Si}$, and $\mathrm{Fe}$. Throughout the range of direct measurements, protons and helium nuclei are the most abundant cosmic rays and thereby present the least statistical errors. In general for such measurements, higher mass particles are less common and the statistical uncertainties are larger. Common spectral features have recently been reported by multiple experiments across all primary particles. The Alpha-Magnetic Spectrometer (AMS-02), Cosmic Ray Energetics and Mass instrument (CREAM), CALorimetric Electron Telescope (CALET), and DArk Matter Particle Explorer (DAMPE) collaborations have all reported spectral hardening starting at $\sim 200 \mathrm{GV}$ ([2] and references therein). Such hardening is modeled as a transition from one accelerator to another, assuming a simple rigidity dependent acceleration limit, to fit the spectral change. To fit the cosmic ray spectra from direct measurement to above knee energies, we adopt this acceleration model by assuming 4 acceleration sources with rigidity dependent upper limits.

In addition to the $200 \mathrm{GV}$ hardening findings, the AMS- 02 collaboration reported that the 8 primary particles can be split into 3 groups due to identical rigidity dependencies. The Proton Group contains only protons, the Helium Group has He, C, O, and Fe, and the Neon Group is composed of $\mathrm{Ne}, \mathrm{Mg}$ and $\mathrm{Si}$. These groupings were used in this analysis' fitting procedure to assume the same spectral index values per group. 
The compiled elemental spectra measurements end at $\sim 10^{5} \mathrm{GeV}$, about an energy decade below the all-particle knee. At higher energies, unfolded Extended Air Shower (EAS) measurements were compiled, spanning from $10^{6}-10^{10} \mathrm{GeV}$. The compiled EAS experiments predict relative elemental abundances by analyzing the distribution of shower maxima in the atmosphere [3]. Such processes rely on the choice of hadronic interaction model and, in practice, only 3 or 4 different primary particles are assumed to perform such calculations. Calculated EAS fluxes were compiled for the Proton and Helium Groups ([4] and references therein). With the lack of compiled EAS data for the Neon Group, we estimated the group's contributions by subtracting the Proton and Helium Group fits from available all-particle data.

\subsection{Spectrum Modeling}

This analysis attempts to explain the knee, as well as other changes in the index of the spectra, as transitions between populations that have a power law spectrum and exponentially decay at a fixed rigidity cutoff value. The flux of each primary particle $j$ is modeled as a function of total energy $E$ shown in Equation (1).

$$
F_{j}(E)=\sum_{i=0}^{3} a_{j, i} E^{\gamma_{j, i}} \times \exp \left(\frac{-E}{Z_{j} * R_{i}}\right)
$$

in which $i$ is the acceleration population, $a_{j, i}$ is the coefficient of the flux, $\gamma_{j, i}$ is the spectral index, $Z_{j}$ is the primary particle charge, and $R_{i}$ is the rigidity dependent cut off, which is chosen for each population. Based on the AMS-02 collaboration's finding on the group behavior, each element in the particle group is fit with the same index. By assuming rigidity cutoffs and identical indices for each particle grouping, the number of overall fitting parameters are reduced, thereby simplifying the model. Note that this model assumes 4 populations that are dominant at different regions of the spectrum: a Population 0 at below knee energies, a Population 1 at around knee energies, and Populations 2 and 3 that correspond to above knee energies. The cutoff rigidity values, found by fitting the data, are given below in Table 1. Three different Population 1 rigidity cutoffs were compared to test how various all-particle knees affect the expected atmospheric neutrino flux. The values of $a_{j, i}$ and $\gamma_{j, i}$ are then fit to the compiled data.

Table 1: Rigidity Cutoffs for all three considered fits

\begin{tabular}{|l||l|l|l|l|}
\hline Case & Pop. 0 Cutoff & Pop. 1 Cutoff & Pop. 2 Cutoff & Pop. 3 Cutoff \\
\hline \hline Case I & $400 \mathrm{GV}$ & $50 \mathrm{TV}$ & $4 \mathrm{PV}$ & $500 \mathrm{PV}$ \\
\hline Case II & $400 \mathrm{GV}$ & $250 \mathrm{TV}$ & $4 \mathrm{PV}$ & $500 \mathrm{PV}$ \\
\hline Case III & $400 \mathrm{GV}$ & $800 \mathrm{TV}$ & $4 \mathrm{PV}$ & $500 \mathrm{PV}$ \\
\hline
\end{tabular}

\subsection{Neutrino Flux Calculation}

Once the cosmic ray spectra were fit, the expected atmospheric muon neutrino flux was calculated. When cosmic rays reach Earth and produce air showers, amongst the many particles created, charged pions and kaons are abundant. These particles then commonly decay into muon 
neutrinos of variable energy depending on the initial energy of the cosmic ray and the subsequent atmospheric interactions. Due to the various production channels of charged pions and kaons, cosmic ray to neutrino flux simulations must be robust to accurately predict resulting atmospheric neutrino fluxes. In this analysis, the Matrix Cascade Equations (MCEq) toolkit was utilized to handle the cosmic ray to atmospheric neutrino flux calculations [5]. MCEq is capable of taking various cosmic ray spectra models, simulating air showers in Earth's atmosphere, and tracking resulting fluxes for $\sim 50$ elements and particles. In this analysis we input the fitted cosmic ray elemental spectra and tracked atmospheric muon neutrino production. There are two main portions of the MCEq simulation that can be altered: the atmospheric density profile and hadronic interaction model. The effect of changing these parameters is shown in Figure 1.

By comparing the solid and dashed lines, it is evident that the choice of atmospheric density profile has very little effect on the resulting data- the largest difference between fluxes was found to be less than $5 \%$. With this in mind, this analysis uses the MSISO0 atmospheric density profile [7]. Of larger consequence is the choice of hadronic interaction model; by comparing the yellow and purple lines in Figure 1, clearly the choice of model has a large effect on resulting fluxes, especially at higher energies. In order to account for these variations, two hadronic interaction models, SYBILL2.3c [7] and QGSJett-II-04 [9], were utilized. These models act at the upper and lower bounds, re-

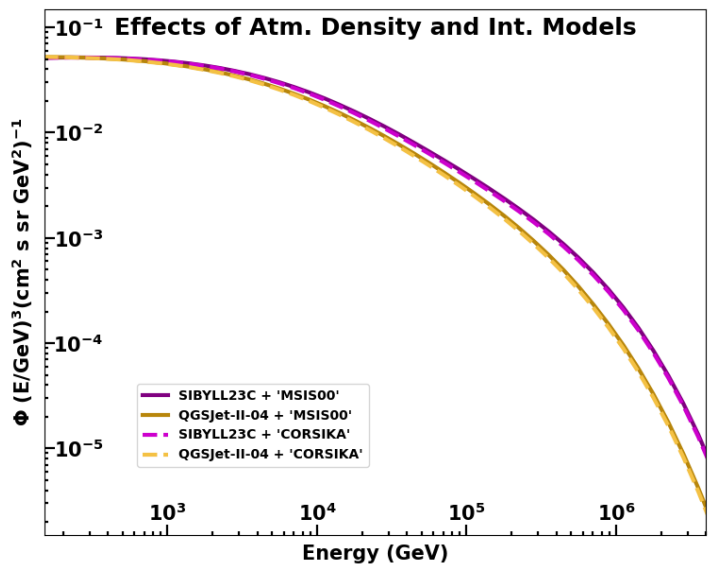

Figure 1: Effects of atmospheric density profile $[6,7]$ (solid vs dashed lines) and hadronic interaction models $[8,9]$ (purple vs yellow lines) spectively, of the possible fluxes. The simulation location was chosen to be the South Pole, and the resulting fluxes were averaged over both zenith angle and season to suppress any fluctuations due to these variables.

\section{Results and Discussion}

The resulting elemental fits for proton, helium, and neon from Case II are shown in Figure 2 as an example of our results. The underlying populations are shown in purple, with Population 0 being the left most curve and Population 3 being the right most. The contributions of these populations are summed to produce the green curve, which is fit to match the compiled elemental data. At the highest energies, the Proton Group and Helium Group are fit to EAS data. For illustration purposes, the expected Neon Group contribution, found by subtracting the Proton and Helium Group fits from all-particle data, is shown by the blue shaded region in the Neon plot. The Neon Group contribution is fit to these region, and is shown by the dashed green line in the plot. The fitted spectral indices for the three elemental groups are shown in Table 2.

The all-particle knee is located at $\sim 10^{6} \mathrm{GeV}$, around which Population 1 begins to drop off as Population 2 starts to dominate. For the low Population 1 cutoff, the overall abundances of elements 
decreased around the knee, requiring Population 2 to be boosted to compensate. The opposite is true for higher rigidity cutoffs; Population 1 is boosted, whereas Population 2 is decreased
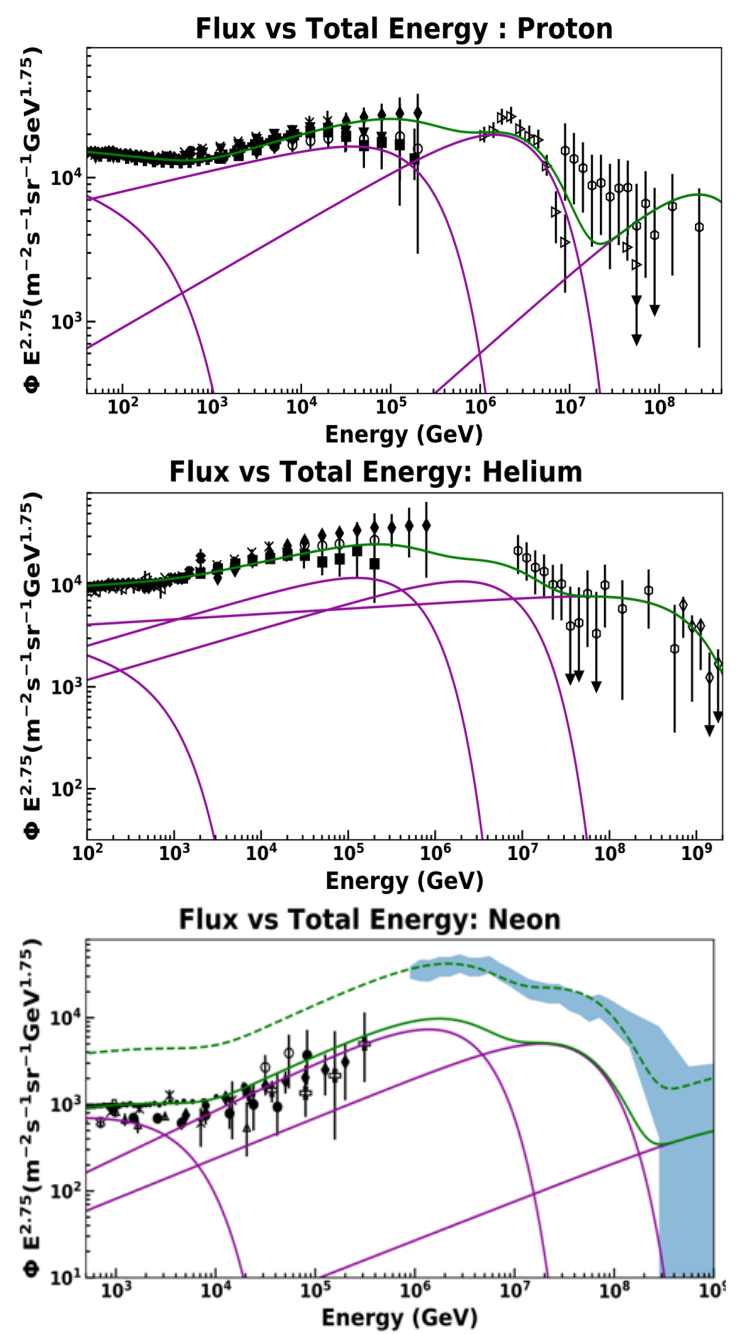

Figure 2: Elemental group fits with underlying population contributions. Direct measurement data points: open thick X's, HEAO-3, open squares, ACE-CRIS, open small circles, AMS-01, thin X's, ATIC-2, open thick pluses, TRACER, open large circles, CREAM1, open left-pointing triangles, BESS, filled thick X's, PAMELA, filled large circles, CREAM-2, filled diamonds, NUCLEON, filled stars, AMS-03, filled small circles, AMS-02, open down-pointing triangles, JACEE, thin pluses, RUNJOB, open up-pointing triangles, CRN, filled squares, CREAM-3, filled downpointing triangles, DAMPE, open stars, LEAP, and filled thick pluses, CALET ([2] and references therein). EAS data points: open right-pointing triangles, KASCADE + QGSJett01, open diamonds, Pierre Auger + QGSJett2.04, and open pentagons, TUNKA + QGSJett01 ([4] and references therein). relative to Figure 2. Thus, Case III results in a higher all-particle flux compared to Case I below the knee, and lower above the knee. Population 3 then begins to dominate above $\sim 10^{8}$ $\mathrm{GeV}$.

The all-particle spectrum was calculated by summing the contributions of each elemental fit, which can be seen in Figure 3 for all three of our cases. Our calculated all-particle spectra for the 3 cases are compared to compiled all-particle data from various experiments ([10, 11] and references therein) and Gaisser's H3a fit [10]. Generally, our results are lower than the all-particle data below $10^{5} \mathrm{GeV}$. Above this energy, Case II and Case III match the expected behavior, and above $10^{6} \mathrm{GeV}$, Case I reflects the data as well. This agreement continues until $10^{8} \mathrm{GeV}$, above which no attempt was made to fit the data in this study. Although additional fitting is necessary to obtain better agreement below $10^{5} \mathrm{GeV}$, the all-particle knee energy regime is well modeled, indicating an investigation into the resulting neutrino fluxes is appropriate.

The resulting neutrino fluxes were calculated from the elemental fits using the MCEq toolkit, the results of which are shown in Figure 3. The predicted fluxes are compared to IceCube's IC-59 [12] (open circles) and IC-79 [1] (filled circles) atmospheric muon neutrino data sets. Note that the IC-79 data diverges from the fits and IC-59 data at $\sim 100 \mathrm{TeV}$. This is because, as discussed, at these energies the astrophysical signal begins to dominate the spectrum, which IC-79 measured. IC-59 was an earlier, incomplete configuration of the IceCube detector, and thus was not sensitive enough to detect this contribution. It should be noted that, roughly, cosmic rays will produce neutrinos about two orders of magnitude lower in energy.

Below $10^{3} \mathrm{GeV}$, all three cases result in 
Table 2: Fitting results of spectral index values for Case II

\begin{tabular}{|l||l|l|l|l|}
\hline Elemental Group & $\gamma_{0}$ & $\gamma_{1}$ & $\gamma_{2}$ & $\gamma_{3}$ \\
\hline \hline Proton Group & -2.95 & -2.60 & -2.39 & -2.2 \\
\hline Helium Group & -2.94 & -2.50 & -2.50 & -2.70 \\
\hline Neon Group & -2.64 & -2.2 & -2.29 & -2 \\
\hline
\end{tabular}

higher atmospheric neutrino flux than the IceCube data. Above $10^{3} \mathrm{GeV}$, all fits are in close agreement with both data sets, and continue to be in close agreement with the IC-59 data above $\sim 10^{5} \mathrm{GeV}$. Generally, between $10^{3}$ and $10^{5} \mathrm{GeV}$, the Case III fit predicts the most atmospheric neutrinos, whereas the Case I fit predicts the least. Above this energy, all three fits predict a similar flux. This is expected; as mentioned before, higher rigidity cutoffs have a boosted Population 1 relative to lower rigidity cutoffs, resulting in higher fluxes around the knee and increased production of atmospheric neutrinos. Above the knee, at $10^{5} \mathrm{GeV}$ in neutrinos, lower rigidity cutoffs require a boosted Population 2 in order to be in agreement with data, resulting in similar atmospheric neutrino fluxes.
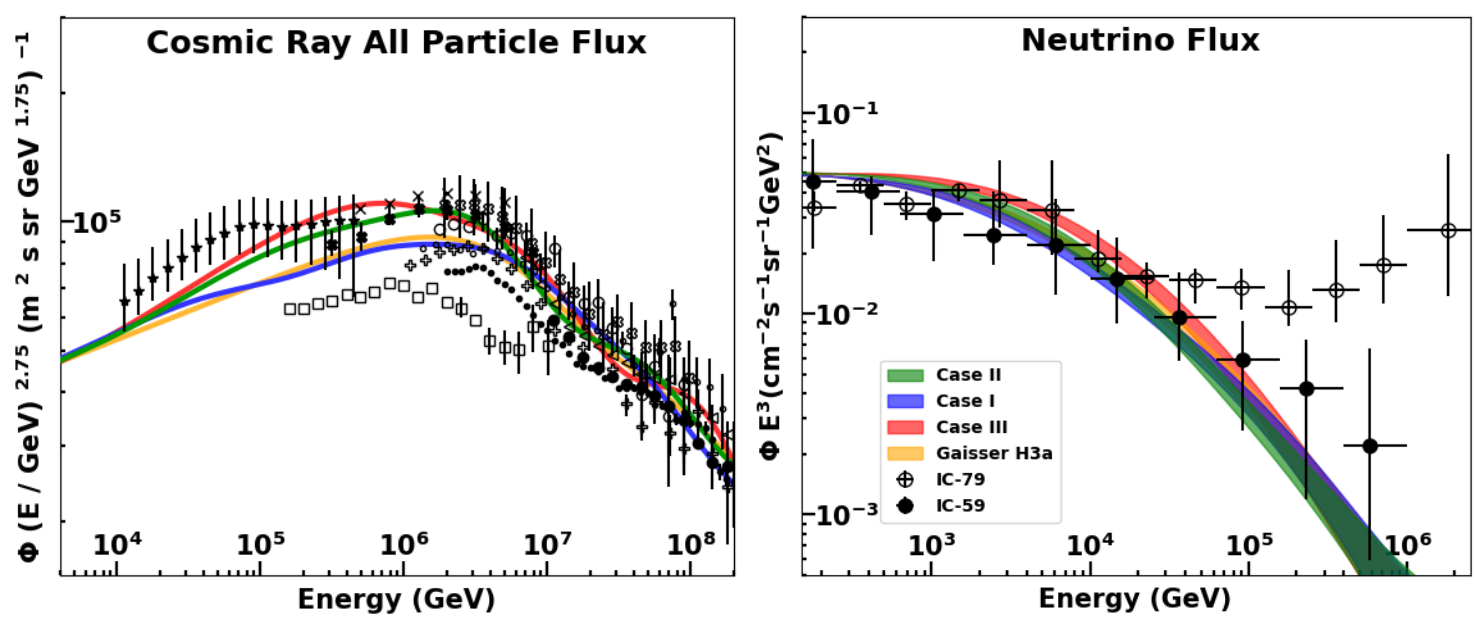

Figure 3: Calculated all-particle (left) and atmospheric neutrino (right) fluxes. For the all-particle data points: open thick X's, IceTop (2016), open squares, CASA-MIA, open small circles, GAMMA, thin X's, HEGRA, open thick pluses, Tibet-III, open large circles, KASCADE, open left-pointing triangles, Tunka, filled large circles, KASCADE-Grande ([10] and references therein), filled stars, HAWC and filled thick X's, IceTop (2020) ([11] and references therein).

One point of interest, when comparing the all-particle cosmic ray flux to the calculated atmospheric neutrino spectra, occurs at $10^{6} \mathrm{GeV}$ in cosmic rays. At about this energy, the Case II fit surpasses the Case III fit in terms of absolute flux. Yet, at the corresponding neutrino energy of $10^{4}$ $\mathrm{GeV}$, no such transition occurs. It is of interest to investigate the contributions of each cosmic ray element to understand this perceived discrepancy. Figure 4 shows the underlying contributions of each element to both spectra. Notice that although proton is not the most abundant element in the cosmic rays flux across the entire spectrum, it still has the largest contribution to the atmospheric neutrino flux across the full range of energies. About $62 \%$ of the neutrino flux arises from parent protons, $21 \%$ from helium, and the remaining percentages from heavier elements. Thus, the 
neutrino flux is especially sensitive to the underlying abundances of the lightest elements.

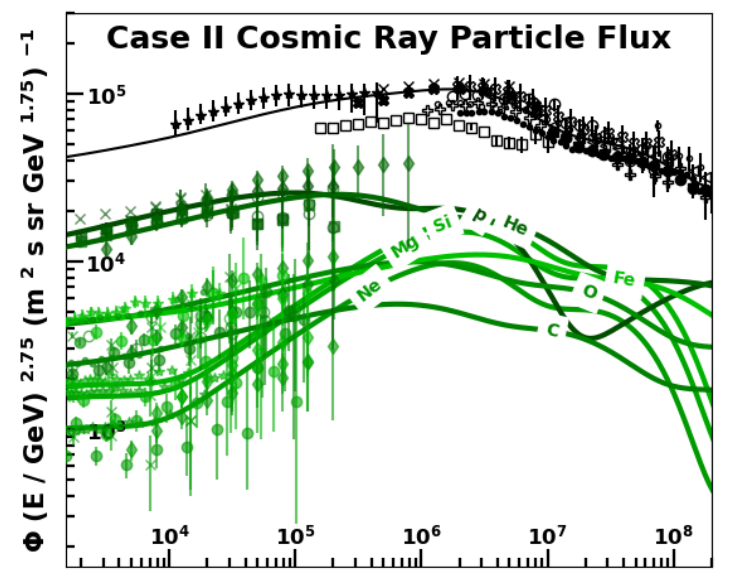

Energy (GeV)

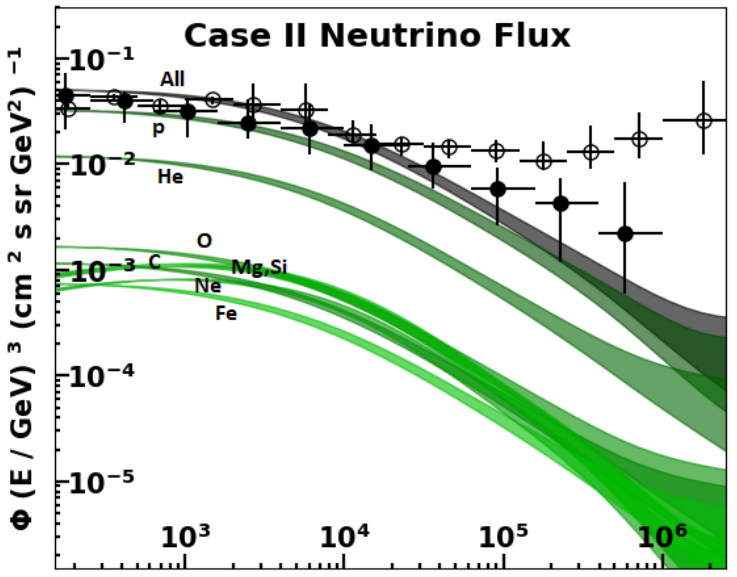

Energy (GeV)

Figure 4: Underlying elemental contributions to the cosmic ray spectrum (left) and neutrino spectrum (right)

By comparing the resulting all particle spectrum and the atmospheric neutrino spectrum across all three fits, some general behaviors are determined. For example, at and below $10^{5} \mathrm{GeV}$, our calculated curves are below the cosmic ray all particle data. However, at the corresponding neutrino energies of $10^{3} \mathrm{GeV}$ and below, our calculated curves are above the neutrino data. This indicates a potential overabundance of light elements, and in general some tension between our cosmic ray fits and the calculated atmospheric neutrino spectra. As more heavy element data become available, further fitting can be explored in order to potentially lower the Proton and Helium Group abundances and increase the Neon Group abundances.

Another general behavior is found when considering the atmospheric neutrino spectra above $10^{5} \mathrm{GeV}$. Note that this range corresponds to cosmic ray energies above $10^{7} \mathrm{GeV}$, which is very well modeled by our fits. Although all three cases predict different underlying spectra in cosmic rays, the resulting atmospheric neutrino spectra above $10^{5} \mathrm{GeV}$ are very similar. All three cases predict fluxes at the lower edge of the IC-59 data range, indicating that the true atmospheric neutrino flux could also lie at the lower error bar edge. Clearly, by uniting both cosmic ray and atmospheric neutrino measurements, conclusions about both spectra can be drawn that otherwise would remain less understood.

\section{Conclusion}

By compiling decades of elemental spectra from direct and unfolded EAS measurements, this analysis was able to fit a 4 population model, assuming different types of sources, to elemental measurements and produce both all-particle and atmospheric muon neutrino spectra. It was found that all three cases are in agreement with all-particle data around and at the knee. Utilizing the MCEq toolkit, the fitted elemental spectra were used to calculate atmospheric neutrino fluxes. It was found that all three cases tended to have higher atmospheric neutrino fluxes compared to the compiled IceCube data below $10^{3} \mathrm{GeV}$, indicating an potential overabundance in light elements. This points to some unresolved tension between cosmic rays and neutrinos that will continue to be explored. Furthermore, only three rigidity cutoffs, representing the range of possible values, were 
explored in this work; a more exhaustive investigation will be carried out to determine the best fit to all the considered data.

\section{References}

[1] M.G. Artsen et al., Measurement of the $v_{\mu}$ energy spectrum with IceCube-79, Eur. Phys. J. C 77, 692 (2017).

[2] E.S. Seo, Advances in-direct measurements of cosmic rays, J. Korean Phys. Soc. 78, 923-931 (2021).

[3] A. Aab et al., Depth of Maximum of Air-Shower Profiles at the Pierre Auger Observatory: Measurements at Energies above $10^{17.8} \mathrm{eV}$, Phys. Rev. D 90, 12, 122005 (2014).

[4] H. Dembinski et al., Data-driven model of the cosmic-ray flux and mass composition from 10 $\mathrm{GeV}$ to $10^{11} \mathrm{GeV}$, in proceedings of PoS(ICRC2017) 301.

[5] A. Feydnitch et al., Calculation of conventional and prompt lepton fluxes at very high energy, EPJ Conf. 99, 08001 (2015).

[6] D. Heck et al., CORSIKA: A Monte Carlo code to simulate extensive air showers, Tech. Rep. FZKA 6019, Karsruhe (1998).

[7] J.M. Picone et al., NRLMSISE-00 empirical model of the atmosphere: Statistical comparisons and scientific issues, J. Geophys. Res. 107(A12), 1468, (2002).

[8] F. Riehn et al., The hadronic interaction model Sibyll 2.3c and Feynman scaling, in proceedings of PoS(ICRC2017) 301.

[9] S. Ostapchenko, Monte Carlo treatment of hadronic interactions in enhanced Pomeron scheme: QGSJET-II model, Phys.Rev. D 83, 014018 (2011).

[10] T.K. Gaisser et al., Cosmic Ray Energy Spectrum from Measurements of Air Showers, Frontiers of Physics 8, 248-259 (2013).

[11] M.G. Aartsen et al. (IceCube Collaboration) Cosmic ray spectrum from $250 \mathrm{TeV}$ to $10 \mathrm{PeV}$ using IceTop Phys. Rev. D 102, 122001 (2020).

[12] M.G. Artsen et al., Development of a general analysis and unfolding scheme and its application to measure the energy spectrum of atmospheric neutrinos with IceCube, Eur. Phys. J. C 75, 116 (2015). 